\title{
An Improved Optimal Control Model of HIV Based on Treatment and Screening
}

\author{
Zhaofu Qun ${ }^{1, a}$ \\ ${ }^{1}$ XianYang Normal University, XianYang ShanXi, 712000, China \\ aemail: fuqunzhao@126.com
}

Keywords: Human Immunodeficiency Virus; Improved Control Model; Optimal Control

\begin{abstract}
In this paper, an optimal control model based on an improved HIV model is considered. Using of condom, screening of unaware infectives and incentive for treatment of the infected are used as the control items. Base on the same objective functional to minimize the unaware individuals and the cost of three kinds control at a given stage, we give four different optimal strategies with four different control suppose. By comparing the optimal strategies and dynamic trend of our model, we find that the dynamic trends are almost the same and the three different controls play different role, but simulation also shows that the screening control of unaware infectives play a relative small role when compare with the control of condom use and incentive control for treatment.
\end{abstract}

\section{Introduction}

Acquired Immunity Deficiency Syndrome(AIDS) is the disease that has affected the whole world in the 20 years since it was first detected. It is caused by Human Immunodeficiency Virus(HIV). 34.3 million People live with HIV infection today and more than 24 million are in the developing world [1].

Mathematical models of transmission dynamics of HIV play an important role in better understanding of epidemiological patterns for disease control as they provide short and long term prediction of HIV and AIDS incidence[2,3,4,5,6,7,8]. On the other hand, optimal control theory has been applied extensively in HIV models [9, 10, 11, 12, 13, 14, 15]. H. R. Joshi [15] built optimal control model about HIV based on an ordinary differential equation. The optimal drug strategies are determined for various stages of initiation of treatment. Later, the HIV models become more and more complicated because more factors were considered, such as activated and resting CD4+cells were contained. Further more, two major categories of anti-retroviral drugs to combat HIV are reverse transcriptase inhibitors (RTIs) and protease inhibitors (PIs), then the RTI control variable and the PI control variable were joined into the system[16]. In paper [8], a HIV model was given as follows:

$$
\left\{\begin{array}{l}
\frac{d S}{d t}=Q_{0}-\beta_{H} S-\mu S \\
\frac{d I_{1}}{d t}=\beta_{H} S-(\theta+\delta+\mu) I_{1}, \\
\frac{d I_{2}}{d t}=\theta I_{1}-(\delta+\mu+\pi) I_{2}, \\
\frac{d H}{d t}=\pi I_{2}-(\sigma \delta+\mu) H \\
\frac{d A}{d t}=\delta I_{1}+\delta I_{2}+\sigma \delta H-(\alpha+\mu) A
\end{array}\right.
$$

where $S(t)$ is the susceptible individuals, the unaware infective individuals is denoted by $I_{1}(t)$, $I_{2}(t)$ represent the aware infective individuals by screening but not be on treatment, the individuals who are on treatment are stood for by $H(t)$ and $A(t)$ represents the AIDS population. 
$\beta_{H}=\frac{\left(1-u_{1}\right)\left(\beta_{1} c_{1} I_{1}+\beta_{2} c_{2} I_{2}+\beta_{3} c_{3} A+\beta_{h} c_{h} \mathrm{H}\right)}{N}$, where $u_{1}\left(0 \leq u_{1} \leq 1\right)$ is the condom use control, $N(t)=S(t)+I_{1}(t)+I_{2}(t)+H(t)+A(t)$ is the number of the total human population at time $t$, the parameters $\beta_{i}(i=1,2,3, h)$ are the probabilities for susceptible individuals with unaware infectives, infectives have been aware, AIDS individuals and HIV positive individuals on treatment respectively. $Q_{0}$ is the constant rate of immigration of the susceptible, $\mu$ represents the natural mortality rate, $\theta$ measures the rate at which unaware infectives are detected by a screening method to become aware infectives, $\pi$ measures the rate at which the already aware infective individuals receive treatment, $\delta$ is the rate by which all the individuals with HIV develop AIDS, $\sigma$ is the modification parameter due to treatment, $\alpha$ is the AIDS related death rate.

Paper [8] pointed that effective control of the disease may be too costly when constant controls are considered as it requires treatment at higher levels for all time and then gave a time dependent controls model is as follows:

$$
\left\{\begin{array}{l}
\frac{d S}{d t}=Q_{0}-\beta_{H} S-\mu S, \\
\frac{d I_{1}}{d t}=\beta_{H} S-\left(u_{2} \theta+\delta+\mu\right) I_{1}, \\
\frac{d I_{2}}{d t}=u_{2} \theta I_{1}-\left(\delta+\mu+u_{3} \pi\right) I_{2}, \\
\frac{d H}{d t}=u_{3} \pi I_{2}-(\sigma \delta+\mu) H, \\
\frac{d A}{d t}=\delta I_{1}+\delta I_{2}+\sigma \delta H-(\alpha+\mu) A .
\end{array}\right.
$$

where $u_{2}\left(0 \leq u_{2} \leq 1\right)$ is the control on screening of unaware infectives, $u_{3}\left(0 \leq u_{3} \leq 1\right)$ is the control on treatment of infectives. Paper [8] also gave some simulations to compare the function of different controls, but $u_{2}=0$ in model (2) means that there is no screening of unaware infectives, and it neglects the rate at which unaware infectives are detected to become aware infectives without screening method. As for $u_{3}$, since $0 \leq u_{3} \leq 1$, it seems unreasonable because the already aware infective individuals have their own right to choose to be treated or not. So in order to find the optimal screening method, and considering the corresponding intervention to treatment, we gave another optimal control model with screening method and intervention to treatment as follows:

$$
\left\{\begin{array}{l}
\frac{d S}{d t}=Q_{0}-\beta_{H} S-\mu S, \\
\frac{d I_{1}}{d t}=\beta_{H} S-\left(\left(1+u_{2}\right) \theta+\delta+\mu\right) I_{1}, \\
\frac{d I_{2}}{d t}=\left(1+u_{2}\right) \theta I_{1}-\left(\delta+\mu+\left(1+u_{3}\right) \pi\right) I_{2}, \\
\frac{d H}{d t}=\left(1+u_{3}\right) \pi I_{2}-\left(\left(1-\varepsilon u_{3}\right) \delta+\mu\right) H, \\
\frac{d A}{d t}=\delta I_{1}+\delta I_{2}+\left(1-\varepsilon u_{3}\right) \delta H-(\alpha+\mu) A .
\end{array}\right.
$$

where $u_{2}$ represents the passive screening, and $\theta$ represents the active screening by individuals, $u_{3}$ represents the incentive control for treatment, we use $\left(1-\varepsilon u_{3}\right) \delta$ to reflect the effect to progression rate of HIV positive individuals on treatment to AIDS population by the control $u_{3}$. This paper intend to search for the optimal control strategy and compare the role of each control.

The organization of the paper is as follows. In section 2, the optimal control problem is proposed, the existence of the optimal control system is proved. Section 3 simulates the different 
cases based on four kinds of control. Section 4 draws the conclusion.

\section{The existence of the optimal control}

Our optimal goal is to minimize the number of unaware infectives and the cost of applying the control $u_{1}, u_{2}$ and $u_{3}$, so we choose the objective functional $J\left(u_{1}, u_{2}, u_{3}\right)$ as follows:

$$
J\left(u_{1}, u_{2}, u_{3}\right)=\min _{u_{1}, u_{2}, u_{3}} \int_{t_{0}}^{t_{f}}\left(a I_{1}+b_{11} u_{1}^{2}+b_{22} u_{2}^{2}+b_{33} u_{3}^{2}\right) d t .
$$

where $U=\left\{\left(u_{1}, u_{2}, u_{3}\right) \mid u_{i}\right.$ measurable, $a_{i} \leq u_{i} \leq b_{i}, t \in\left[t_{0}, t_{f}\right]$, for $\left.i=1,2,3\right\}$ is the control set. which $a_{i}$ and $b_{i}(i=1,2,3)$ stand for the minimum and maximum effect. The term $a$ represents the weight of $I_{1}$ and the positive constants $b_{11}, b_{22}, b_{33}$ takes into account financial constraints. We will prove the existence of $\left(u_{1}, u_{2}, u_{3}\right)$ satisfy that: $\min _{\left(u_{1}, u_{2}, u_{3}\right)} J\left(u_{1}, u_{2}, u_{3}\right)=J\left(u_{1}^{*}, u_{2}^{*}, u_{3}^{*}\right)$.

To use an existence result, we must check the following properties [17]:

1 . The set of controls and corresponding state variables is non-empty.

2. The control $U$ set is convex and closed.

3. The state system is bounded in the state and control variables.

4. The integrand of the objective functional is concave on $U$.

5. There exists constants $\eta_{1}, \eta_{2}>0$, and $\beta>1$ such that the integrand $L\left(I_{1}, u_{1}, u_{2}, u_{3}\right)$ of the objective functional satisfies:

$$
L\left(I_{1}, u_{1}, u_{2}, u_{3}\right) \geq \eta_{1}\left(\left|u_{1}\right|^{2}+\left|u_{2}\right|^{2}+\left|u_{3}\right|^{2}\right)^{\beta / 2}-\eta_{2} .
$$

In order to verify these conditions, we use a result by [18] to give the existence of solutions of model (3) with bounded coefficients, which gives condition 1. We note that the solutions are bounded. Our control set satisfies condition 2. Equation (3) satisfies condition 3 is easily to verified, using the boundedness of the solutions.

Note that the integrand of our objective functional is concave. Also we have the last condition needed: $a I_{1}+b_{11} u_{1}^{2}+b_{22} u_{2}^{2}+b_{33} u_{3}^{2} \geq \eta_{1}\left(\left|u_{1}\right|^{2}+\left|u_{2}\right|^{2}+\left|u_{3}\right|^{2}\right)-\eta_{2}$.

where $\eta_{1}$ depends on the upper bound on $I_{1}$; and $\eta_{2} \geq 0$ since $b_{11}, b_{22}, b_{33}>0$. We conclude there exists an optimal control pair.

The Hamiltonian function, defined by using the dynamic constraint (3) and the Lagrangian $L=a I_{1}+b_{1} u_{1}^{2}+b_{2} u_{2}^{2}+b_{3} u_{3}^{2}$ is as follows:

$$
\begin{aligned}
& H^{*}=L+\lambda^{T} f=a I_{1}+b_{11} u_{1}^{2}+b_{22} u_{2}^{2}+b_{33} u_{3}^{2}+\lambda_{1}\left(Q_{0}-\beta_{H} S-\mu S\right)+\lambda_{2}\left(\beta_{H} S-\left(\left(1+u_{2}\right) \theta_{1}\right.\right. \\
& \left.+\delta+\mu) I_{1}\right)+\lambda_{3}\left(\left(1+u_{2}\right) \theta_{1} I_{1}-\left(\delta+\mu+u_{3} \pi\right) I_{2}\right)+\lambda_{4}\left(u_{3} \pi I_{2}-\left(\left(1-\varepsilon u_{3}\right) \delta+\mu\right) H\right) \\
& +\lambda_{5}\left(\delta I_{1}+\delta I_{2}+\left(1-\varepsilon u_{3}\right) \delta H-(\alpha+\mu) A\right)
\end{aligned}
$$

Using Pontryagin's minimum principle, the necessary conditions (in terms of the Hamiltonian) for $U^{*}$ to be an optimal control is as following:

$$
1^{\circ}\left\{\begin{array}{l}
\frac{d S}{d t}=Q_{0}-\beta_{H} S-\mu S, \\
\frac{d I_{1}}{d t}=\beta_{H} S-\left(\left(1+u_{2}\right) \theta+\delta+\mu\right) I_{1}, \\
\frac{d I_{2}}{d t}=\left(1+u_{2}\right) \theta I_{1}-\left(\delta+\mu+\left(1+u_{3}\right) \pi\right) I_{2}, \\
\frac{d H}{d t}=\left(1+u_{3}\right) \pi I_{2}-\left(\left(1-\varepsilon u_{3}\right) \delta+\mu\right) H, \\
\frac{d A}{d t}=\delta I_{1}+\delta I_{2}+\left(1-\varepsilon u_{3}\right) \delta H-(\alpha+\mu) A .
\end{array}\right.
$$


$2^{\circ} S\left(t_{0}\right)=S_{0} ; I_{1}\left(t_{0}\right)=I_{10} ; I_{2}\left(t_{0}\right)=I_{20} ; H\left(t_{0}\right)=H_{0} ; A\left(t_{0}\right)=A_{0}$.

$3^{\circ}\left[\begin{array}{c}\dot{\lambda}_{1} \\ \dot{\lambda}_{2} \\ \dot{\lambda}_{3} \\ \dot{\lambda}_{4} \\ \dot{\lambda}_{5}\end{array}\right]=-\left[\begin{array}{lllll}\frac{\partial H^{*}}{\partial S} & \frac{\partial H^{*}}{\partial I_{1}} & \frac{\partial H^{*}}{\partial I_{2}} & \frac{\partial H^{*}}{\partial H} & \frac{\partial H^{*}}{\partial A}\end{array}\right]^{T}$,

$4^{\circ} \quad \lambda_{i}\left(t_{f}\right)=0, \quad i=1,2,3,4,5$.

$5^{\circ}\left[\begin{array}{l}\frac{\partial H^{*}}{\partial u_{1}} \\ \frac{\partial H^{*}}{\partial u_{2}} \\ \frac{\partial H^{*}}{\partial u_{3}}\end{array}\right]=\left[\begin{array}{l}2 b_{11} u_{1}+\left(\lambda_{1}-\lambda_{2}\right) \frac{\beta_{1} c_{1} S I_{1}+\beta_{1} c_{1} S I_{2}+\beta_{1} c_{1} S A+\beta_{1} c_{1} S H}{S+I_{1}+I_{2}+H+A} \\ 2 b_{22} u_{2}-\theta_{1} I_{1}\left(\lambda_{2}-\lambda_{3}\right) \\ 2 b_{33} u_{3}-\pi I_{3} \lambda_{3}+\pi I_{3} \lambda_{4}+\varepsilon \delta H \lambda_{4}-\varepsilon \delta H \lambda_{5}\end{array}\right]=0$.

So we get the optimal control:

$$
\left\{\begin{array}{l}
u_{1}^{*}=\max \left\{a_{1}, \min \left\{\left(\lambda_{2}-\lambda_{1}\right) \frac{\beta_{1} c_{1} S I_{1}+\beta_{1} c_{1} S I_{2}+\beta_{1} c_{1} S A+\beta_{1} c_{1} S H}{2 b_{11}\left(S+I_{1}+I_{2}+H+A\right)}, b_{1}\right\}\right\}, \\
u_{2}^{*}=\max \left\{a_{2}, \min \left\{\frac{\theta_{1} I_{1}\left(\lambda_{2}-\lambda_{3}\right)}{2 b_{22}}, b_{2}\right\}\right\}, \\
u_{3}^{*}=\max \left\{a_{3}, \min \left\{\frac{\pi I_{2}\left(-\lambda_{3}+\lambda_{4}\right)-\delta H\left(\lambda_{5}-\lambda_{4}\right)}{2 b_{33}}, b_{3}\right\}\right\} .
\end{array}\right.
$$

The optimal control system thus, is a coupled forward state equation and a backward adjoint equation, along with the regular control. This problem, being nonlinear and coupled in nature, needs to be solved using concurrent and iterative numerical procedures. In this paper, the optimal strategy is simulated by solving the state and adjoint systems and the transversality conditions based on Runge-Kutta fourth order scheme and the steepest gradient method [19].

\section{Simulation and discussions}

Table 1 : The parameter values we used

\begin{tabular}{|lcc|}
\hline Parameters & Value & Reference \\
\hline$\beta_{1}$ & 0.8 & {$[8]$} \\
$\beta_{2}$ & 0.55 & {$[8]$} \\
$\beta_{3}$ & 0.32 & {$[8]$} \\
$\beta_{h}$ & 0.15 & {$[8]$} \\
$\mu$ & 0.2 & {$[8]$} \\
$\alpha$ & 1 & {$[8]$} \\
$Q_{0}$ & 2000 & {$[8]$} \\
$\delta$ & 0.1 & {$[8]$} \\
$\theta$ & 0.002 & {$[8]$} \\
$\pi$ & 0.66 & {$[8]$} \\
$\sigma$ & 0.002 & {$[8]$} \\
$c_{1}$ & 0.1 & {$[8]$} \\
$c_{2}$ & 0.1 & {$[8]$} \\
$c_{3}$ & 0.1 & {$[8]$} \\
$\varepsilon$ & 0.002 & Assumed \\
\hline
\end{tabular}


In this section, we simulate the optimal control of model (3) and compare the four cases of condom use, screening of unaware infectives and incentive control for treatment on the transmission dynamics of the disease. The parameter values we used are given in Table 1.

The cost coefficients $a=800, b_{11}=35, b_{22}=95, b_{33}=175$ and the initial condition is taken to be $S(0)=800, I_{1}(0)=100, I_{2}(0)=100, H(0)=100, A(0)=100$. Efficacy $u_{1}, u_{2}, u_{3}$ can theoretically lie between 0 and 1 . The minimum effect $a_{i}(i=1,2,3)$ is set to be 0 , which corresponds to no control function. However, the maximum effect is taken to be less than 1 , for the control can't be completely effective and, so the maximum effect $b_{i}(i=1,2,3)$ is set to be 0.98 .

We investigate and compare numerical results in the following scenario:

Case 1 The three kinds of control efforts on condom use $\left(u_{1}\right)$, screening of unaware infectives $\left(u_{2}\right)$ and treatment $\left(u_{3}\right)$ are made to be optimized, the simulation results are shown in Figure 1 and Figure 2.
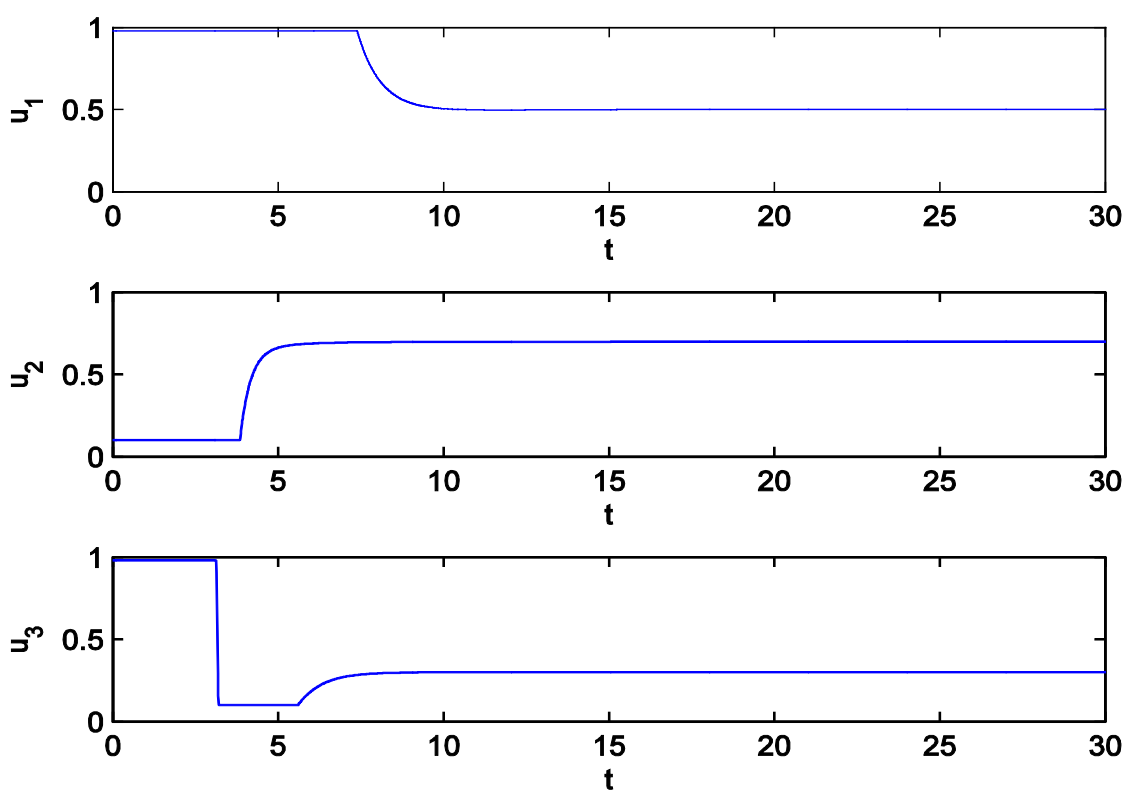

Figure 1 The optimal control strategy of $u_{1}, u_{2}, u_{3}$


Figure 2 The comparison between the optimal control and no control

We use red curve to represent the dynamic trend with optimal control and blue curve to 
represent the dynamic trend with no control. From Figure 2 we know that the optimal control can make the susceptible individuals far more than that with no control, while the other four kinds of individuals in our model much less than that with no control, which show that the HIV has been effectively controlled based on the our optimal control strategy. But since our optimal goal is to minimize the number of unaware infectives and the cost of applying the control, so when the condom use and Incentives for treatment keep the maximum level at the beginning stage, the level of screening of unaware infectives should be kept at a low level.

In the following part, we choose one of the three controls $\left(u_{1}, u_{2}, u_{3}\right)$ to be zero to simulate the corresponding optimal control and compare their effect on the dynamics trend of the five kinds of individuals in our model.

Case 2: The treatment control $\left(u_{3}\right)$ is set to zero and optimal control result of $u_{1}$ and $u_{2}$ are given in Figure 3. The dynamics trend of the five kinds of individuals with and without control are given in Figure 4.
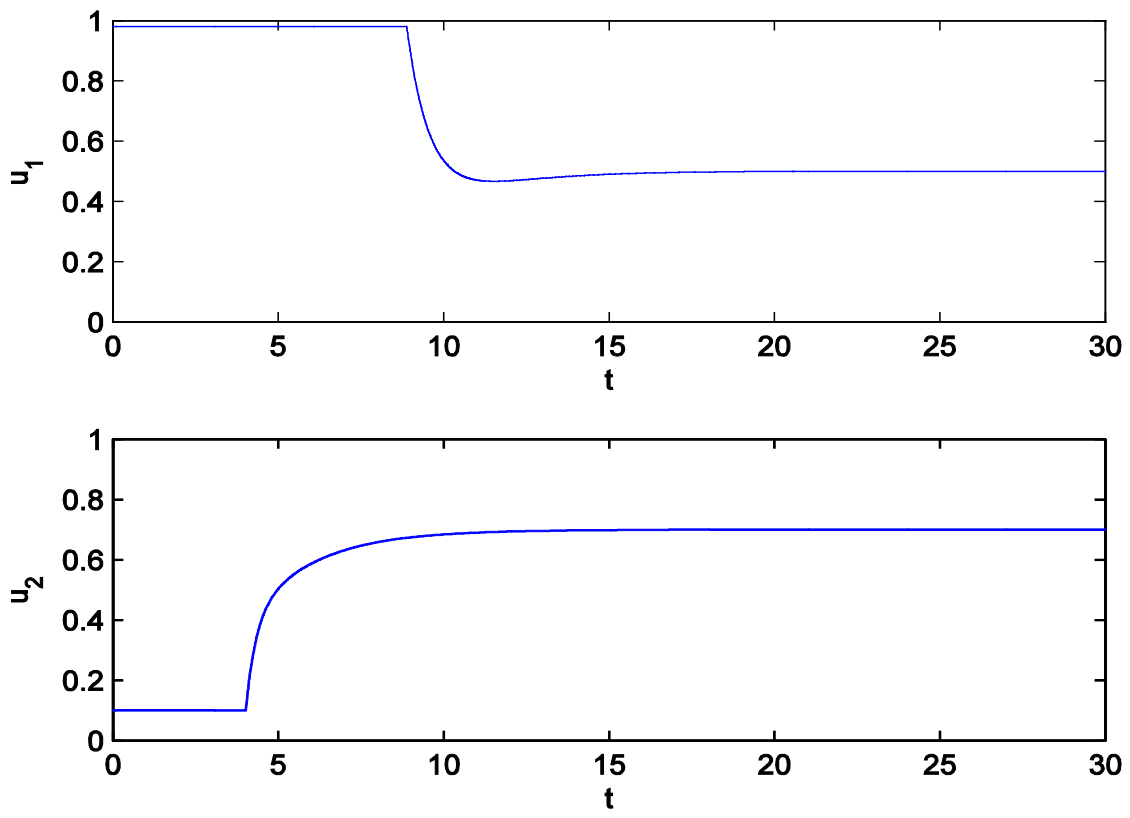

Figure 3 The optimal control strategy of $u_{1}, u_{2}$


Figure 4 The comparison between the optimal control $u_{1}, u_{2}$ and no control. 
Case 3: The control on screening of unaware infectives $\left(u_{2}\right)$ is set to zero and the optimal control result of $u_{1}$ and $u_{3}$ are given in Figure 5. The dynamics trend of the five kinds of individuals with and without control are given in Figure 6.
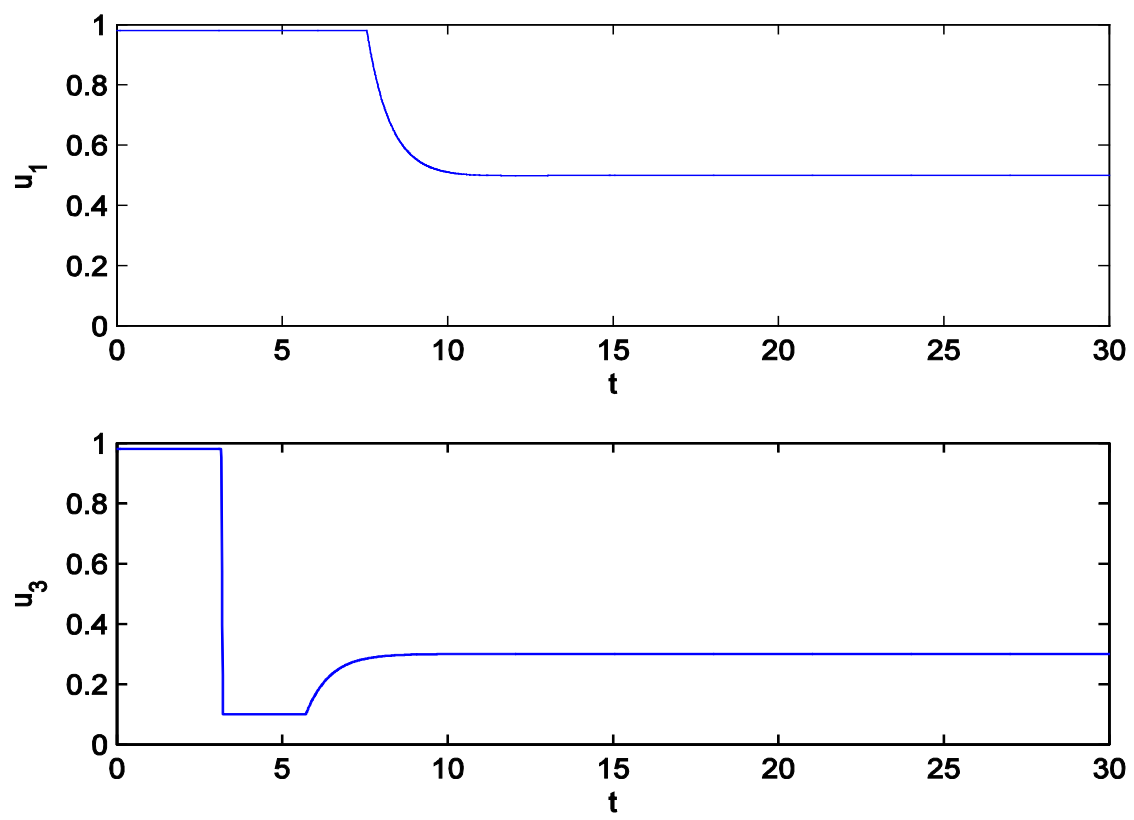

Figure 5 The optimal control strategy of $u_{1}, u_{3}$
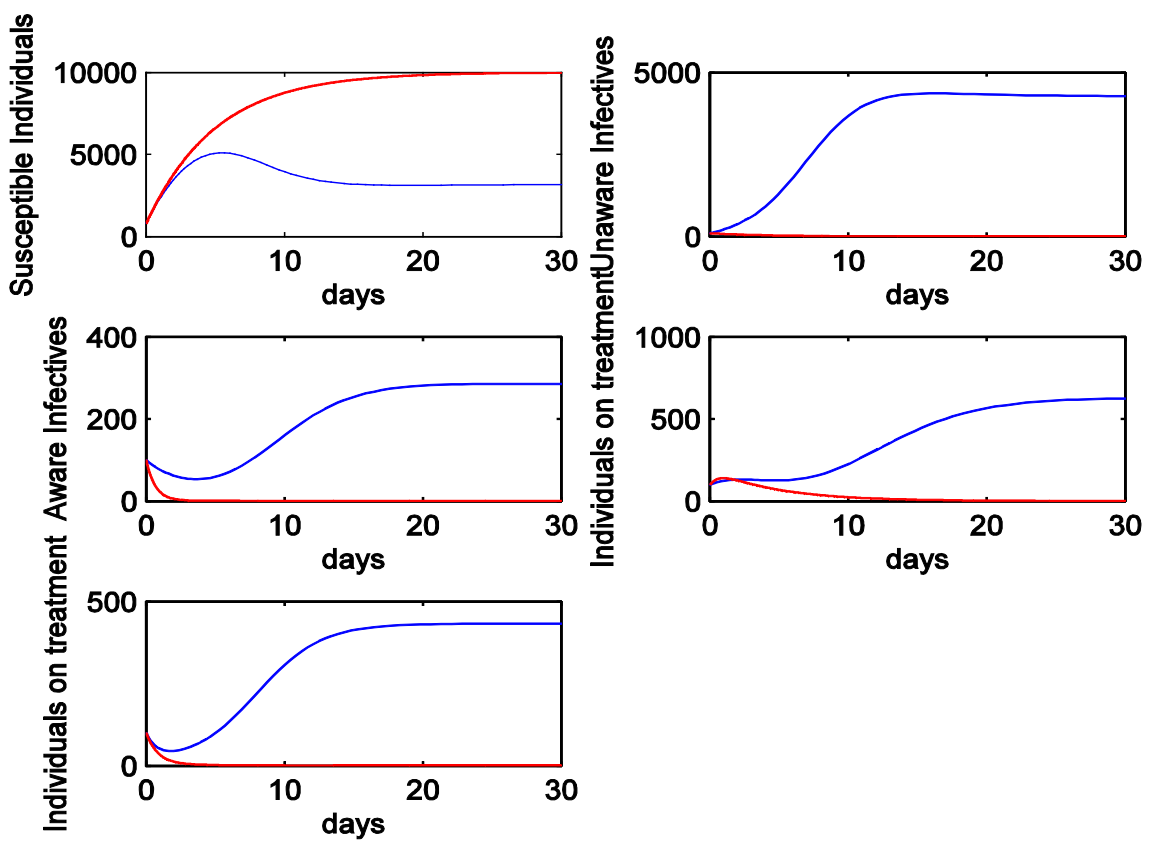

Figure 6 The comparison between the optimal control $u_{1}, u_{3}$ and no control.

Case 4: The control on condom use $\left(u_{1}\right)$ is set to be zero and the optimal control result of $u_{2}$ and $u_{3}$ are given in Figure 7. The dynamics trend of the five kinds of individuals with and without control are given in Figure 8.

By comparing Figure 1 with Figure 3, we find that when incentives for treatment is zero(Case 2), the control of condom use should take longer time to keep maximum effect. But form Figure 1 and Figure 5, no obvious difference can be seen when screening of unaware infectives is zero(Case 3), which shows that the screening of unaware infectives $\left(u_{2}\right)$ has almost no impact on $u_{1}$ and $u_{3}$. Similarly, when the control of condom use is zero (Case 4), the screening of unaware infectives and 
drug treatment should take longer time than Case 1 . As to the dynamics trend, there are no obvious differences within Figure 2, Figure 4, Figure 6 and Figure 8. From the above analysis, we found that the screening control of unaware infectives play a relative small role, the more effective control should be the control of condom use and incentive control for treatment.


Figure 7 The optimal control strategy of $u_{2}, u_{3}$.
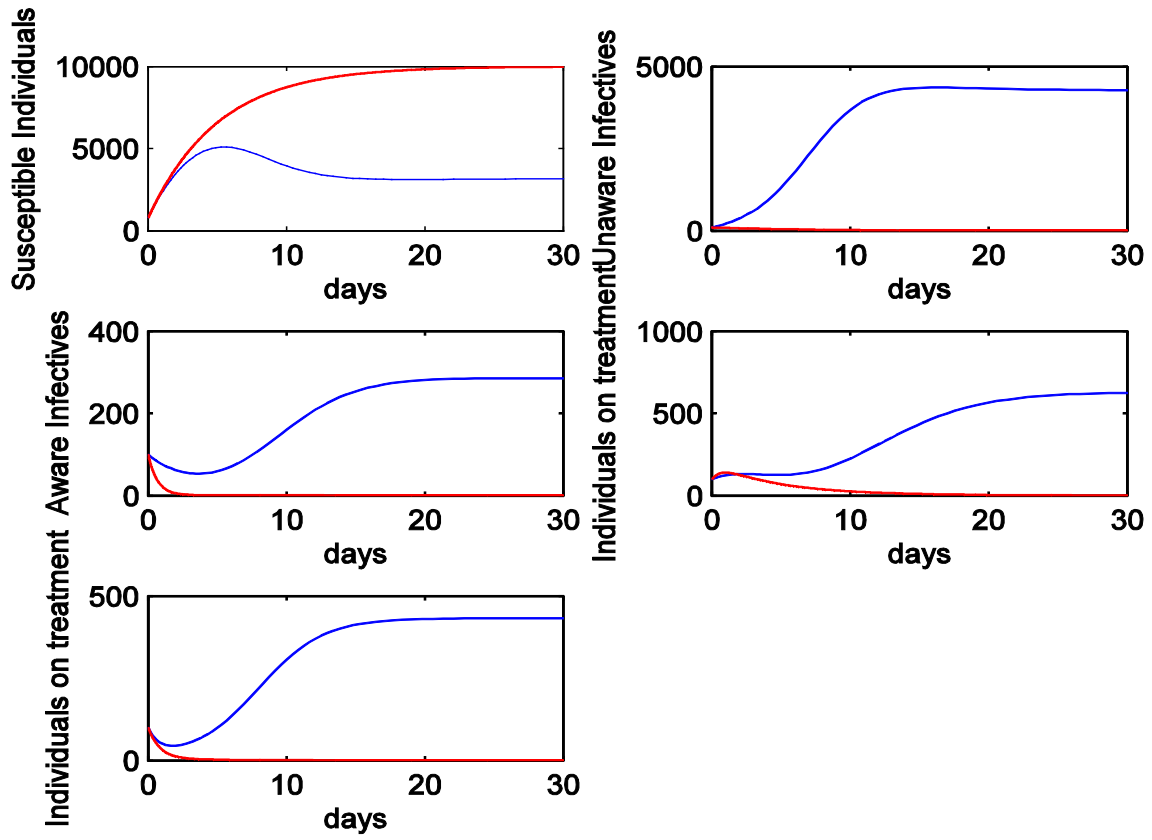

Figure 8 The comparison between the optimal control $u_{2}, u_{3}$ and no control.

\section{Conclusion}

In this paper, we modified the optimal control for HIV model. The conditions for optimal control of the disease with effective use of condoms, incentive control for treatment and screening of infectives were analyzed. We find that if the objective functions are same, the dynamic trends of the five kinds of individuals in our model are almost the same whether or not one of the control is set to be zero. By setting one of the three controls to be zero, we found that the three different controls play different role, when incentives for treatment is zero(Case 2), the control of condom 
use should take longer time to keep maximum effect, when the control of condom use is zero (Case 4), the screening of unaware infectives and drug treatment should take longer time, but simulation showed that the screening control of unaware infectives play a relative small role when compare with the control of condom use and incentive control for treatment, so in order to minimize the cost of applying the control, the screening control of unaware infectives can be weakened while the other two controls should be encouraged.

It should be pointed that the true situation must be more complex and varied than what we have described, but the analytical results could give structures for treatment that could prove foundational for future practice. We believe that the analysis and simulation presented in this paper, can play some role in developing an improved HIV treatment regimen.

\section{Acknowledgments}

We would like to thank the anonymous referees which have improved the quality of our study. We also would like to thank the support of the special scientific research program of Shanxi Provincial Education Department (NO. 13JK0368).

\section{References}

[1] M. Roshanfekr, M. H. Farahi, R. Rahbarian (2014), A different approach of optimal control on an HIV immunology model, Ain Shams Engineering Journal, 1(5):213 - 219.

[2] D. Kirschner (1996), Using mathematics to understand HIV immune dynamics. Am. Math. Soc. 43:191-202.

[3] A.Tripathi (2007) , R. Naresh, D. Sharma, Modelling the effect of screening of unaware infectives on the spread of HIV infection, Applied Mathematics and Computation 184:1053-1068.

[4] R.M. Anderson (1986), G.F. Medly, R.M. May, A.M. Johnson A.M., A preliminary study of the transmission dynamics of the Human Immunodeficiency Virus (HIV), the causative agent of AIDS, IMA J. Math. Appl. Med. Biol. 3:229-263.

[5] E. A. Hernandez-Vargasa, R. H. Middleton (2013), Modeling the three stages in HIV infection, Journal of Theoretical Biology, 320:33-40.

[6] J. Karrakchou, M. Rachik, S. Gourari (2006), Optimal control and infectiology: Application to an HIV/AIDS model, Applied Mathematics and Computation 177:807-818.

[7] J. M. Orellana (2011), Optimal drug scheduling for HIV therapy efficiency improvement, Biomedical Signal Processing and Control, 6: 379-386.

[8] K.O. Okosun, O.D. Makinde, I. Takaidza (2013), Impact of optimal control on the treatment of HIV/AIDS and screening of unaware infectives, Applied Mathematical Modelling, 37:3802-3820.

[9] J.A.M. Felippe de Souza, M.A.L. Caetano, T. Yoneyama (2000), Optimal control applied to the antiviral treatment of AIDS, in: Proceedings of the 39th IEEE Conference on Decision and Control, Sydney, Australia,4839-4844.

[10] M.J. Mhawej, C.H. Moog, F. Biaforeb, B.F. Cecile (2010), Control of the HIV infection and drug Dosage, Biomedical Signal Processing and Control, 5:45-52.

[11] M. Barao, J.M. Lemos (2007), Nonlinear control of HIV-1 infection with a singular perturbation model, Biomedical Signal Processing and Control, 2:248-257.

[12] V. Costanza, P.S. Rivadeneira, F.L. Biafore (2013), Optimizing thymic recovery in HIV patients through multidrug therapies, Biomedical Signal Processing and Control, 8:90-97.

[13] J.M. Orellana (2011), Optimal drug scheduling for HIV therapy efficiency improvement, Biomedical Signal Processing and Control, 6;379-386. 
[14] H.D. Kwon (2007), Optimal treatment strategies derived from a HIV model with drug-resistant mutants. Applied Mathematics and Computation, 188:1193-1204.

[15] H.R. Joshi (2002), Optimal control of an HIV immunology model, optimal control application and methods, 23:199-213.

[16] J. Karrakchoua, M. Rachikb, S. Gouraria (2006), Optimal control and infectiology: Application to an HIV/AIDS model, Applied Mathematics and Computation, 177:807-818.

[17] D.L. Lukes (1982), Differential Equations: Classical to Controlled, Mathematics in Science and Engineering, Academic Press, New York, 162.

[18] Karrakchoua J., Rachikb M., Gouraria S.(2006), Optimal control and infectiology: Application to an HIV/AIDS model, Applied Mathematics and Computation, 177:807-818.

[19] Pachpute G., Chakrabarty S.P. (2013), Dynamics of hepatitis C under optimal therapy and sampling based analysis. Communications in Nonlinear Science and Numerical Simulation, 18 (8): 2202-2212. 